\title{
Mathematical modeling of drop mixing in a slit-type microchannel
}

\author{
K Handique ${ }^{1}$ and M A Burns ${ }^{2,3}$ \\ ${ }^{1}$ Handy Lab, Inc., 3310 Plymouth Road, Ann Arbor, MI 48104, USA \\ ${ }^{2}$ Department of Chemical Engineering, The University of Michigan, Ann Arbor, MI 48109, \\ USA \\ E-mail: maburns@umich.edu
}

Received 9 November 2000, in final form 30 May 2001

Published 9 August 2001

Online at stacks.iop.org/JMM/11/548

\begin{abstract}
Fast solute mixing can be achieved in a microchannel by rapid unidirectional displacement of a discrete liquid drop. The recirculation streamlines created within the liquid during the drop's motion cause the solute to interlayer across the channel depth, provided the interlayer diffusion of the solute is small. Uniform interlayering appears when the drop is displaced by more than three drop lengths in a slit-type microchannel, thereby reducing the solute diffusion distances to a fraction of the channel depth. By fabricating the microchannel to a depth of less than $50 \mu \mathrm{m}$ even large molecules with a low diffusivity $\left(D<10^{-8} \mathrm{~cm}^{2} \mathrm{~s}^{-1}\right.$ ) can be mixed in seconds. The above strategy is shown by modeling the mixing of solutes present in a drop moving in a slit-type microchannel.
\end{abstract}

\section{Introduction}

Over the last few years, numerous works have reported mixing of solutes in microchannels [1-8]. These mixing studies were motivated by the evolving interest in the miniaturization of bench-scale biochemical or chemical processes into sub-microliter or nanoliter systems. The cross-sectional dimensions associated with these miniaturized systems are of the order of micrometers to millimeters [9]. As diffusion is predominant on these length scales, the focus in the design of mixers has been to utilize mixing by molecular diffusion.

A variety of mixers have been designed for continuousflow systems [1-3,5-8], where two liquid streams are made to focus through a channel such that the liquids are mixed during the residence time in the channel. For a given velocity of the fluid, the residence time of the liquid is increased by increasing the length of the channel so as to ensure complete mixing. In some designs the mixing channel is branched into multiple narrower channels so as to ensure mixing in a shorter residence time [1].

As opposed to continuous-flow systems, mixing in systems handling discrete liquid drops [10-16] can be augmented by convective flow. The batch nature of such systems allows these mixers to have a flow-independent residence time. Moreover the recirculation streamlines of

3 Author to whom correspondence should be addressed. liquid in a moving discrete drop [17] allows convective mixing of solutes in addition to molecular diffusion. This recirculation effect has been alluded to in the works of Anderson et al $[14,15]$, Burns et al [10,11], Sammarco [12] and Hosokowa et al [16], and mixing experiments of low molecular weight solutes (diffusivity on the order of $10^{-5} \mathrm{~cm}^{2} \mathrm{~s}^{-1}$ ) have been reported $[15,16]$.

In this paper we performed detailed modeling calculations exploring the effect of velocity, channel dimension and solute diffusivity on the mixing of solutes in a discrete drop for two limiting cases: when convection dominates over diffusion and when diffusion dominates over convection. The discrete drop is assumed to be moved in a slit-type microchannel. Based on these analyses, a mixing strategy is outlined.

\section{Recirculating streamlines in a moving discrete drop}

The liquid inside a discrete drop moving in a microchannel recirculates within itself [17]. As the drop moves in a channel, liquid moves from the center of the plug and adheres to the walls at the front end of the drop. At the receding end of the drop, liquid moves from the wall to the center of the drop. This exchange of liquid at the boundaries and the continuity of liquid within the drop give rise to closed streamlines within a moving discrete drop. 


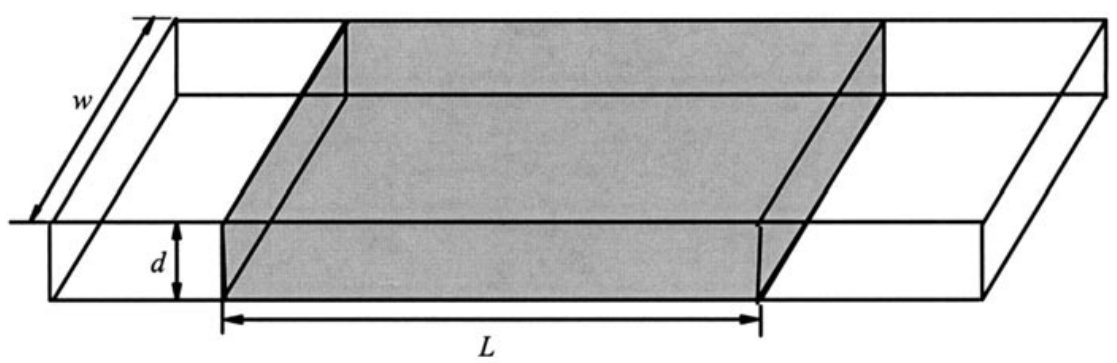

(a)

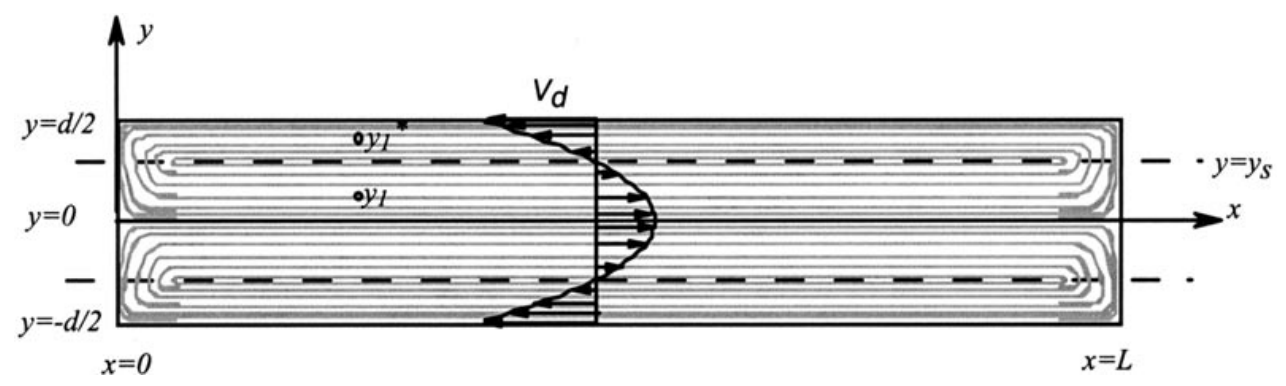

(b)

Figure 1. (a) A discrete drop placed in a slit-type microchannel, where the width of the channel is very large compared to the depth of the channel. (b) Recirculation streamlines present in a drop moving at a constant uniform velocity in a slit-type channel. The frame of reference is moving at the average velocity of the drop $\left(V_{d}\right)$. Liquid in the middle of the drop moves to the leading end of the drop and then towards the channel wall. At the receding end of the drop, liquid moves from the wall towards the center of the drop.

Considerable insight into the mixing of discrete plugs can be obtained by studying the motion of fluid elements in the discrete drop. For computational ease we study the streamlines inside a long drop moving in a slit-type (width $(w) \gg$ depth $(d)$ ) microchannel (figure 1(a)). The physical properties of the liquid (viscosity and density) are assumed to be uniform and constant over the whole liquid. Any surface tension effect on the streamlines is also neglected as the length of the drop is very long compared to the depth of the channel.

The velocity of fluid elements $\left(V_{r e l}\right)$ with respect to the drop moving at a constant velocity $\left(V_{d}\right)$ can be estimated from Hagen-Poisseulli's law [18] as

$$
V_{r e l}(y)=0.5 V_{d}\left(1-3\left(\frac{y}{d / 2}\right)^{2}\right)
$$

where $V_{\text {rel }}$ is the velocity of a streamline at a distance $y$ from the centerline axis. The relative velocity of the streamline $\left(V_{r e l}\right)$ drops to zero at $y=y_{s}=0.577(d / 2)$ (stagnation line). A liquid element moving on its streamline at position $y$ appears at a corresponding streamline at position $y^{*}$ when it reaches the end of the drop. The streamline pairs $\left(y\right.$ and $\left.y^{*}\right)$ can be related to each other by performing a volumetric flow balance around the stagnation line [12] and are given by the following expression

$$
\frac{y^{*}}{d / 2}=-\frac{y}{d}+\frac{\left[4-3\left(\frac{2 y}{d}\right)^{2}\right]^{1 / 2}}{2} .
$$

Figure 1(b) gives a representative plot of the streamlines associated with a moving drop. Using equations (1) and (2), the instantaneous position of any fluid element can be determined for a given average velocity of the drop.

\section{Mixing of solutes in a moving discrete drop}

Consider a soluble substance of a given diffusivity $(D)$ to be non-uniformly dispersed in the combined drop before it is displaced (figure 2(a)). Upon displacing the combined drop by a certain drop length, the solute can either be transported convectively or diffusively to a region of different concentration. The time required for complete mixing (uniform concentration) will therefore depend on the size and velocity of the drop, as well as the diffusivity of the solute.

In order to estimate the concentration profile of the solute, the following coupled convective and diffusive solute transport equation [19] may be solved in conjunction with the velocity profile given in equation (1)

$$
\frac{\partial c}{\partial t}+V_{r e l} \frac{\partial c}{\partial x}=D \frac{\partial^{2} c}{\partial x^{2}}+D \frac{\partial^{2} c}{\partial y^{2}} .
$$

As the concentration depends on two space dimensions as well as velocity, equation (3) would most likely be solved numerically using such techniques as the finite element method.

Although analytical expressions do not exist for the concentration profile, mixing times can easily be estimated for the two following limiting cases: (1) convective transport is very fast compared to diffusive transport and (2) convective transport is very slow compared to diffusive transport. The first case allows one to solve the convection-only equation prior to solving the diffusion-only equation, while the second case allows the coupling of the drop velocity and diffusivity into a single parameter called the dispersion coefficient [20] to describe the mixing process. These two cases are discussed below. 


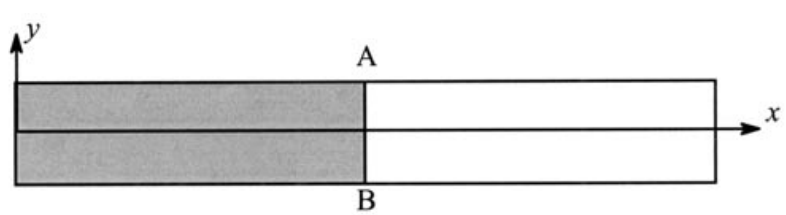

(a)

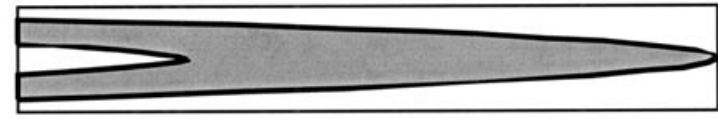

(b)

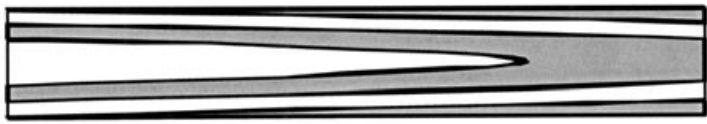

(c)

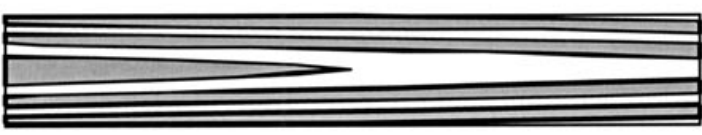

(d)

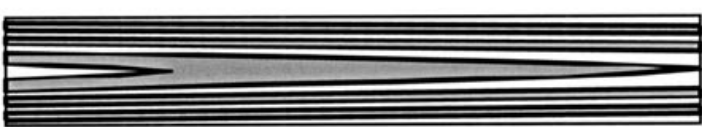

(e)

Figure 2. Effect of streamlines on the distribution of immiscible liquids. (a) Two equal-sized drops are placed end-to-end at $t=0$, (b) The combined drop is moved by a drop length, causing the receding drop to interlayer between the leading drop. Further interlayering is caused after the drop is moved by (c) two drop lengths, (d) three drop lengths and (e) four drop lengths.

\subsection{Case 1. Fast convection compared to diffusion: interlayering}

The conditions for convection to be fast compared to diffusion implies that the time necessary for convective transport $\left(t_{\text {conv }}\right)$ is small compared with the time necessary for diffusive transport $\left(t_{\text {diff }}\right)$. The convective transport timescale, $t_{\text {conv }}$, is on the order of the time required to displace the discrete drop by one drop length. Therefore

$$
t_{\text {conv }} \sim \frac{L}{V_{d}} .
$$

The diffusion timescale, $t_{\text {diff }}$, is the time required for any direct variation of concentration along the depth of the channel to be eliminated. The diffusion timescale can be estimated by solving equation (3) neglecting any diffusion in the $x$-direction and assuming no convective transport $\left(V_{r e l}=0\right)$. For a slittype microchannel, $t_{\text {diff }}$ is given by

$$
t_{\text {diff }} \sim \frac{d^{2}}{\pi^{2} D}
$$

Note that the term $\pi^{2}$ appears in the denominator of (5) because of the nature of the time-dependent concentration profile of the solute in a slit-type channel. The expression of $t_{\text {diff }}$ in
(5) is evaluated using the same method as is described for a cylindrical system by Taylor [21].

As $t_{\text {conv }}$ must be less than $t_{\text {diff }}$, for convection-controlled mixing, the following condition derived using equations (4) and (5) has to be satisfied

$$
V_{d} \gg V_{c}
$$

where $V_{c}$ is the critical interlayering velocity during by the following relation:

$$
V_{c}=\pi^{2}\left(\frac{L}{d}\right) \frac{D}{d} .
$$

The critical interlayering velocity is defined as the velocity of the drop at which the diffusive timescale is equal to the convective timescale (i.e. $t_{\text {conv }} \sim t_{\text {diff }}$ ). As seen in equation (7), the critical interlayering velocity is directly proportional to the solute diffusivity, drop length and inversely proportional to the square of the channel depth (figure 3). Therefore, the critical interlayering velocity can be decreased by reducing the drop length or increasing the channel depth for a solute of specific diffusivity. For channels deeper than $50 \mu \mathrm{m}$ and diffusivity lower than $10^{-6} \mathrm{~cm}^{2} \mathrm{~s}^{-1}$, the critical interlayering velocity for a $2.5 \mathrm{~mm}$ long drop is less than $1 \mathrm{~mm} \mathrm{~s}^{-1}$ (figure 2). Moving the drop at a velocity higher than the critical velocity enables one to operate in the convection-dominated regime.

As the drop is displaced rapidly from its initial position, recirculation is created in the liquid. Because of the non-uniform velocity profile across the channel depth (equation (1)), the fluid elements are non-uniformly displaced with respect to each other, thereby increasing the intermaterial area [22, 23]. The instantaneous value of the intermaterial area can be determined by tracking the position of various points lying on the segment $\mathrm{AB}$ (figure 2(a)) at a particular time and connecting them with a continuous curve (figures 2(b)-(e)). The distribution of liquid is determined as a function of the drop lengths moved and is plotted in figure 2. The liquid stretches and folds across the length of the drop as the drop is unidirectionally displaced. Note that when the intermaterial curve reaches one end of the drop, it appears at its mirror streamline $\left(y^{*}\right)$ at the same end of the drop.

The degree of interlayering of the two liquid concentrations can be measured by calculating the striation length, $s$. The striation length is the average distance between two intermaterial surfaces (figure 4). The striation length can be related to the intermaterial area per unit volume, $a_{v}$, by the following relationship [22, 23]

$$
s \approx \frac{1}{a_{v}}
$$

The intermaterial area per unit volume can be calculated, knowing the length of instantaneous positions of the intermaterial area at various times. As can be seen in figure 5, moving the drop by more than three drop lengths reduces the striation length to below $15 \%$ of the channel depth. Since interlayering starts to appear as soon as the drop is moved, the distribution of the two concentrations is relatively mixed across the length of the drop even after the motion of a single drop length (figure 5). For a drop moved by three drop lengths, the cross-section-averaged concentration of the solute at a 


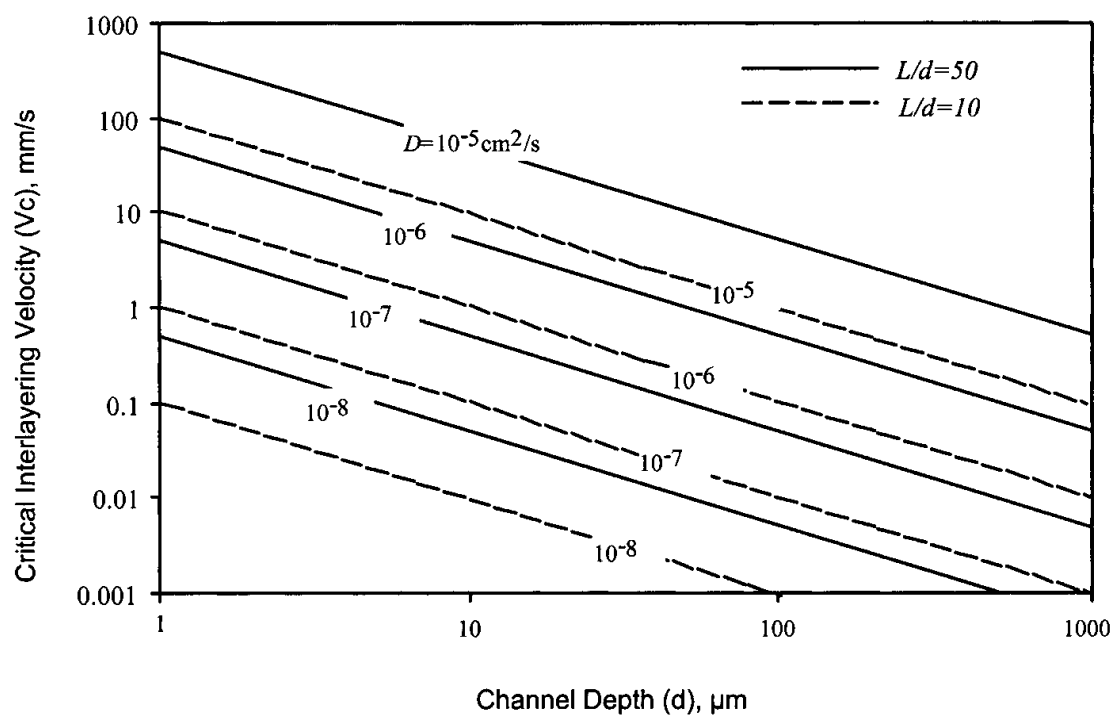

Figure 3. Critical interlayering velocity. The figure plots the velocity at which the effect of convective transport on mixing is the same as that due to diffusion. For mixing to be convective dominated, the velocity should be higher than the critical interlayering velocity.

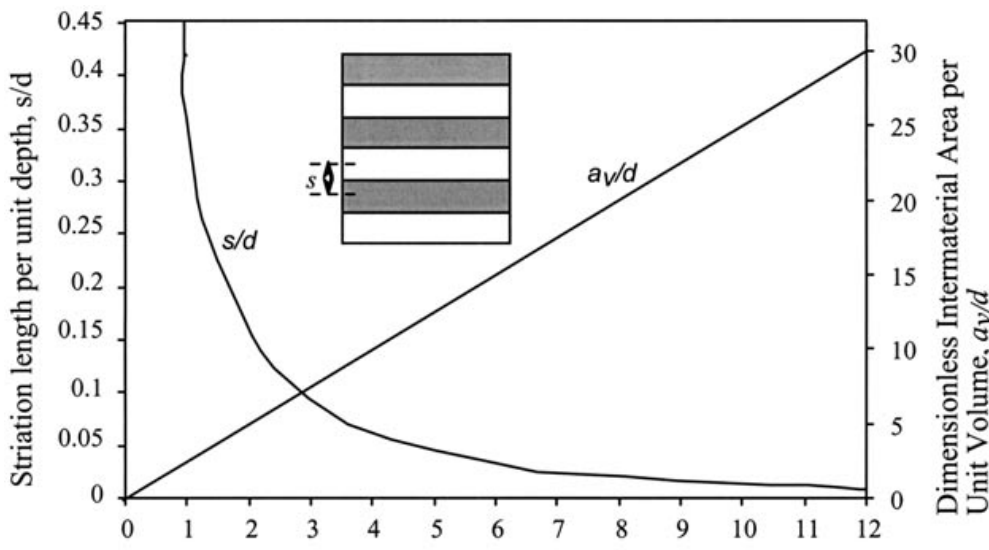

Number of Drop Lengths Moved

Figure 4. The dimensionless striation length $(s / d)$ and the dimensionless intermaterial area per unit volume are plotted as functions of the displacement of a discrete drop. Note, as the drop is moved by three drop lengths, the average striation length decreases to around $15 \%$ of the channel depth (i.e., $s=0.15 d$ ).

particular position is found to be at least $85 \%$ of the volumeaverage concentration over the combined drop.

Once the two liquid concentrations are interlayered, the drop is stopped to let the solute mixed by diffusion. The solute has to diffuse by only the striation length $(s=0.15 d)$ in the $y$-direction to complete the mixing process. The time required for a certain degree of molecular mixing can be estimated by solving for the diffusion equation

$$
\frac{\partial c}{\partial t}=D \frac{\partial^{2} c}{\partial y^{2}} .
$$

Assuming no diffusion of molecules prior to interlayering and a uniform striation length (figure 6(a)), we have the following initial conditions

$$
\begin{array}{cl}
c=c_{\text {max }} & 0 \leqslant y \leqslant \frac{s}{2} \\
c=0 & \frac{s}{2}<y \leqslant s .
\end{array}
$$

As the problem is periodic, we have the following boundary conditions

$$
\frac{\partial c}{\partial y}=0 \quad \text { at } y=0 \text { and } y=s .
$$

The solution for equations (9)-(12) is a series solution and is given by the following equation [24]:

$$
\begin{aligned}
\psi= & \frac{c}{c_{\text {max }}}=1+\sum_{\substack{n=1 \\
n \text { is odd }}}^{\infty}(-1)^{(n-1) / 2} \frac{2}{n \pi} \\
& \times \exp \left(-\frac{t}{\tau} n^{2} \pi^{2}\right) \cos \left(n \pi \frac{y}{s}\right)
\end{aligned}
$$

where $\tau=s^{2} / D$. Figure 6 plots the concentration profile as a function of distance for different times. Based on figure 6 , the time required for at least $90 \%$ mixing $(\psi<0.45)$ to occur is given by $0.35 \tau$. As $s=0.15 d$, the post-interlayering time 


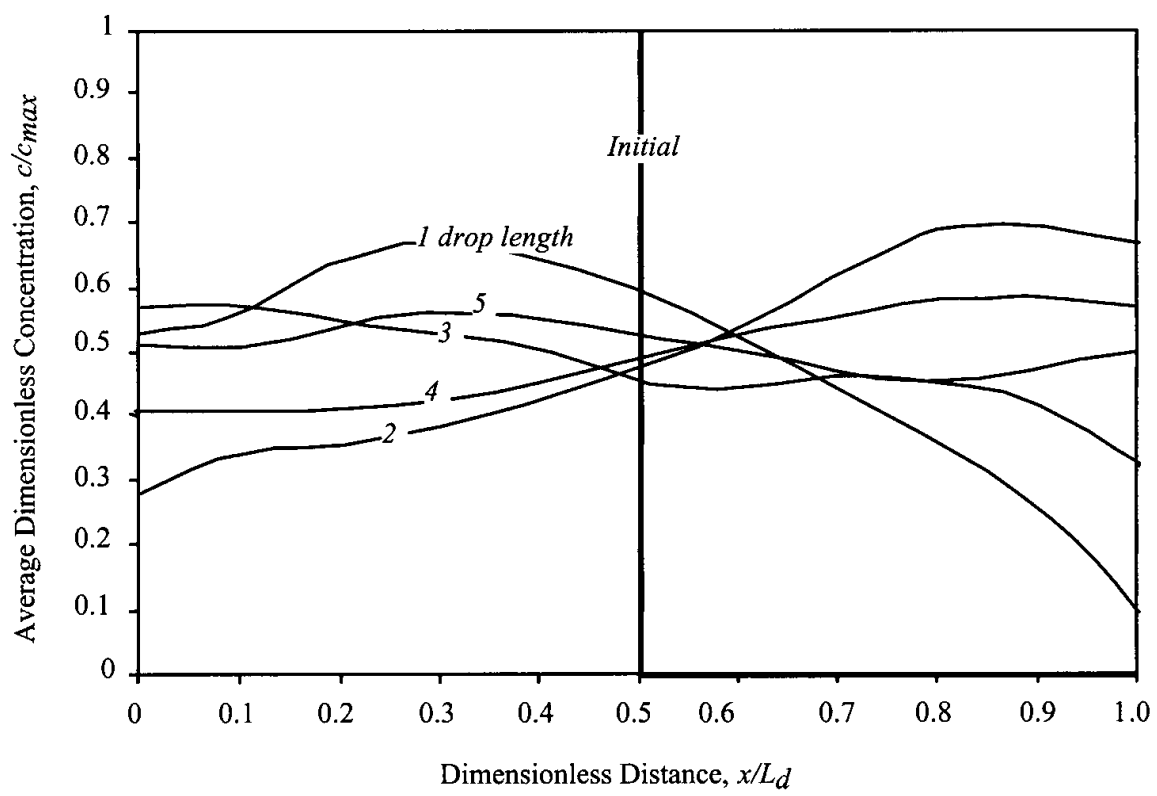

Figure 5. The vertical averaged axial concentration of the solute is plotted for different drop displacements. As the drop is moved by three or more drop lengths, the average axial concentration of liquid is at least $80 \%$ of the volume-averaged concentration of the combined drop.

required for $90 \%$ mixing is given by

$$
t_{\text {diff, }, \text { int }}=0.35 x \frac{(0.15 d)^{2}}{D}=0.008 \frac{d^{2}}{D} .
$$

Figure 7 plots the post-interlayering mixing time as a function of channel depth for different solute diffusivities. Even slowly diffusive molecules can be mixed in a few seconds after interlayering in channels shallower than $50 \mu \mathrm{m}$.

The total mixing times $\left(t_{m i x}\right)$ is given by the sum of the time required to move the drop by three drop lengths and the time required to complete diffusion across the channel depth after interlayering. Therefore, $t_{m i x}$ is given by the following relationship

$$
t_{m i x}=\frac{3 L}{v_{d}}+0.008 \frac{d^{2}}{D} .
$$

As $V_{d} \gg V_{c}$ the drop velocity can also be written in terms of the critical interlayering velocity as $f_{d} V_{c}$, where $f_{d} \gg 1$. The mixing time can now also be written as

$$
t_{\text {mix }}=\frac{3 L}{f_{d} V_{c}}+0.008 \frac{d^{2}}{D}=\left(\frac{0.3}{f_{d}}+0.008\right) \frac{d^{2}}{D} \quad f_{d} \gg 1
$$

If $f_{d}$ is 100 ,

$$
t_{m i x} \approx 0.01 \frac{d^{2}}{D} .
$$

Equation (17) predicts that mixing is only slightly longer than shown in figure $7\left(t_{m i x}=0.008 d^{2} / D\right)$. Therefore mixing can be accomplished for essentially any solute in seconds in the case of convection-dominated mixing.

\subsection{Case 2. Fast diffusion compared to convection: Taylor dispersion}

If the drop velocity is small compared to the critical interlayering velocity (i.e. $V_{d} \ll V_{c}$ ), diffusion dominates convection and interlayering of the concentrations will not take place. This condition leads to diffusion-dominating dispersive mixing in the drop. The mixing due to dispersion can be written in the following form:

$$
\frac{\partial C}{\partial t}=D_{\text {disp }} \frac{\partial^{2} C}{\partial x^{2}}
$$

where $D_{\text {disp }}$ is the dispersion coefficient. The derivation of equation (18) from equation (3) is similar to that outlined in Taylor [21] and Cussler [22]. For a slit-type channel, $D_{d i s p}$ is given by the following relationship:

$$
D_{d i s p}=\frac{d^{2} V_{d}^{2}}{210 D} .
$$

Assuming the drop velocity to be a fraction $\left(f_{d}, 0<f_{d} \ll 1\right)$ of the critical interlayering velocity, we have $V_{d}=f_{d} V_{c}$, where $V_{c}$ is given by equation (7). Therefore, the dispersion coefficient can be written as

$$
D_{\text {disp }}=\frac{d^{2} f_{d}^{2} V_{c}^{2}}{210 D}=\left(\frac{\pi^{4} f_{d}^{2}}{210}\right) \frac{L^{2} D}{d^{2}} .
$$

The solutions for equation (18) is similar to that given by equation (9), except that equation (18) is solved for the $x$ direction and $s$ is replaced by $L$ in equations (10)-(13). Therefore, $90 \%$ of the mixing can be achieved in a time $t_{m i x}$, given by

$$
t_{\text {mix }}=0.35 \tau=\frac{0.35 L^{2}}{D_{\text {disp }}}=\frac{0.754}{f_{d}^{2}} \frac{d^{2}}{D} \quad 0<f_{d} \ll 1
$$

or, if $f_{d}$ is 0.1 ,

$$
t_{m i x} \approx 100 \frac{d^{2}}{D} \text {. }
$$

Comparing equation (22) with (17), we see that the time required to mix after rapid interlayering is four orders of magnitude lower than by dispersion mixing. Moreover, the 


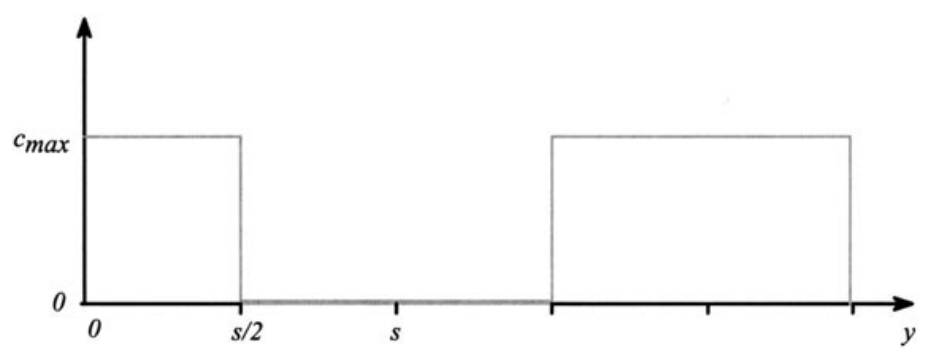

(a)

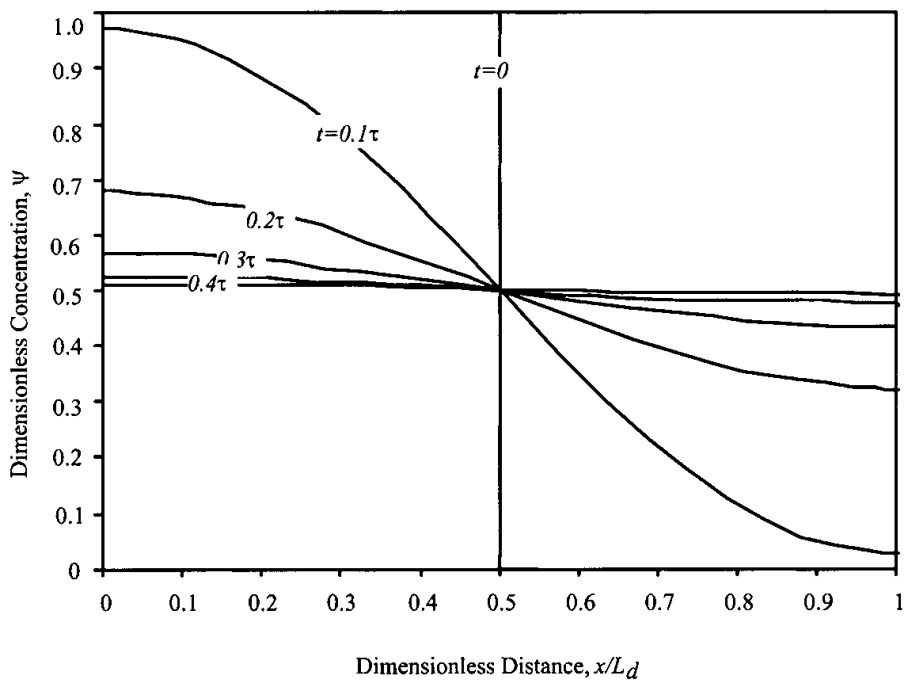

(b)

Figure 6. (a) The initial distribution of concentrations after uniform interlayering. (b) The decay of a square pulse of concentration with respect to time over distance. Almost $90 \%$ mixing is achieved in a time equal to $0.35 \tau$, where $\tau$ is given by $s^{2} / D$.

displacement required for dispersion mixing is much larger $\left(V_{d} \times t_{\text {mix }}=7.44 L / f_{d} \approx 100\right.$ drop lengths $)$ as compared to that required for interlayering mixing (equal to three drop lengths). As the number of displacements required for dispersive mixing is quite high, the drop may be moved back and forth to complete mixing in a limited channel length. Note that this back-and-forth mixing is only valid in the dispersive mixing case since the dispersion coefficient is independent of direction. If back-and-forth motion is used in the case where convection dominates diffusion, no interlayering and hence no mixing is obtained, i.e. the drop is interlayered in one direction and then reset to the original state when reversed.

\subsection{Interlaying versus Taylor dispersion}

The mixing time calculated for both the limiting cases is a function of the ratios $V_{d} / V_{c}$ and $d^{2} / D$. Using equations (16) and (21), we plot the dimensionless mixing time, $\tau_{m i x}$, as a function of the dimensionless drop velocity $\left(V_{d} / V_{c}\right)$, where

$$
\tau_{m i x}=\frac{t_{m i x} D}{d^{2}}
$$

or

$$
t_{m i x}=\tau_{m i x} \frac{d^{2}}{D}
$$

Figure 8 shows a plot of $\tau_{\text {mix }}$ versus $V_{d} / V_{c}$ for both limiting cases. For large values of $V_{d} / V_{c}$ (say $V_{d} / V_{c}>10$ ), mixing occurs by interlayering and equation (16) is valid. For small values of $V_{d} / V_{c}$ (say $V_{d} / V_{c}<0.1$ ), mixing occurs by

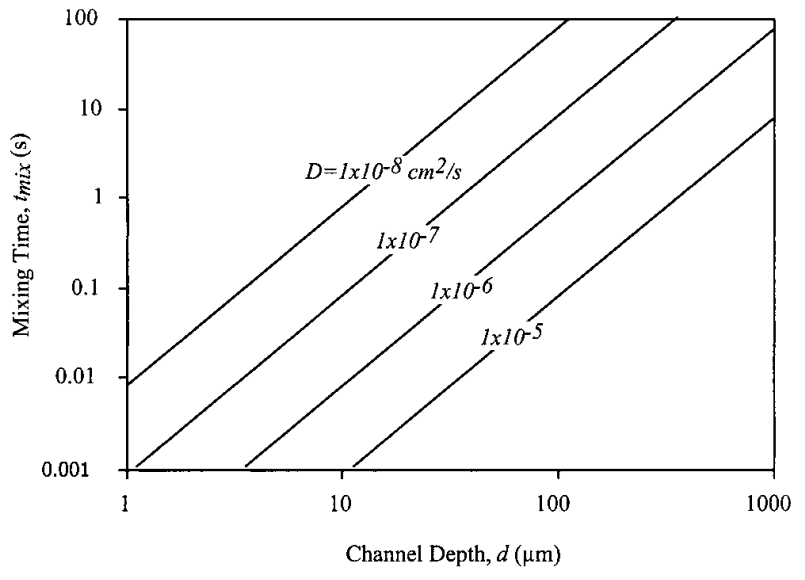

Figure 7. The post-interlayering mixing time required for diffusion mixing across the channel depth. The solute is uniformly interlayered by rapidly displacing the drop from its initial position by three drop lengths.

dispersion and equation (21) is valid. For values of $V_{d} / V_{c} \approx 1$, neither solution presented in this paper is valid. However, both solutions converge to approximately the same value.

\section{Conclusions}

Fast solute mixing can be achieved in a microchannel by rapid unidirectional displacement of a discrete liquid drop. We have examined two limiting cases: fast convection compared to 


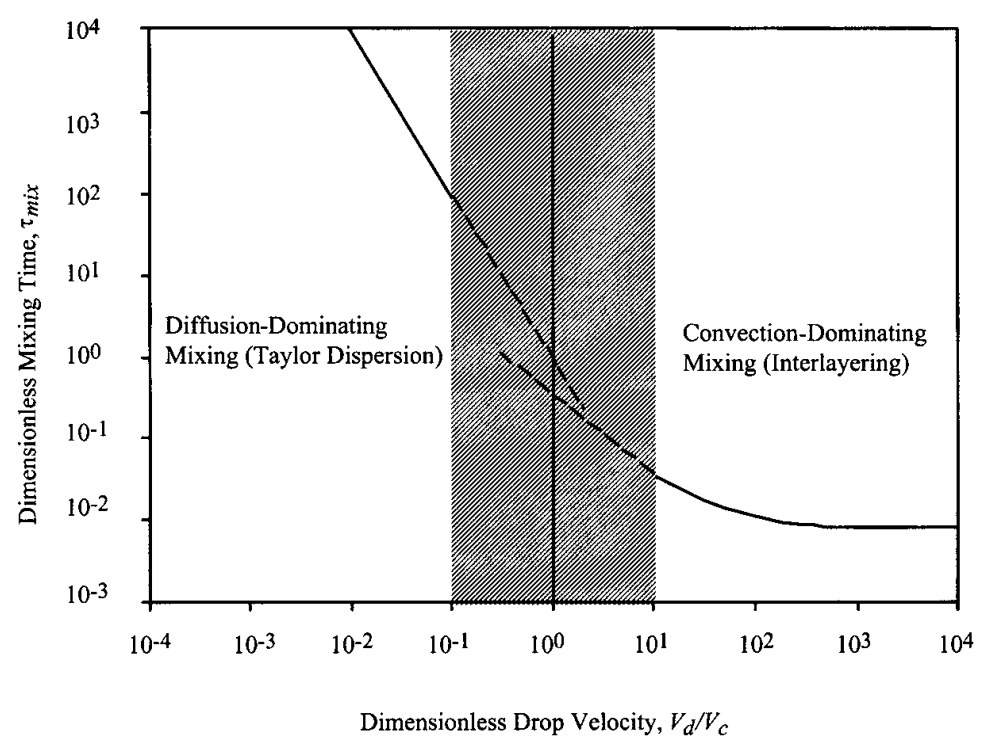

Figure 8. Estimation of mixing time as a function of dimensionless drop velocity. For high drop velocities, convection dominates and the mixing time can be estimated using equation (16), while for low values of the drop velocity, diffusion dominates and the mixing time can be estimated using equation (21). For $V_{d} \sim V_{c}$, an average of the two limiting cases may be used as an estimate of the mixing time.

diffusion and fast diffusion compared to convection. Although the complicated intermediate regime was not examined (the shaded area in figure 8), both solutions converged to approximately the same value, indicating that this analysis can be used to estimate mixing in that region as well. Comparing the two cases, it is clear that the interlayering technique results in the shortest mixing times. Also, back-and-forth mixing can only be used for dispersive mixing; back-and-forth motion for interlayering will result in no mixing.

The technique for calculating the mixing time involves calculating the critical interlayering velocity $\left(V_{c}\right)$ using figure 3 or equation (7) for a specific solute (diffusivity, $D$ ), microchannel (depth, $d$ ) and drop length $(L)$. The discrete drop is displaced by three drop lengths at a velocity of $10 V_{c}$ or more and then stopped. Mixing for that system will be complete in a time that can be estimated using figure 8 or equation (16). Furthermore, to mix a solution containing multiple solutes with widely varying diffusivities, the critical interlayering velocity may be calculated based on the diffusivity of the smallest molecule and the post-interlayering mixing time estimated based on the diffusivity of the largest molecule.

\section{Acknowledgments}

The authors would like to acknowledge the support of the National Institutes of Health under grants NIH-R01-HG01044 and NIH-R01-HG01406 and the Rackham Graduate School at the University of Michigan, Ann Arbor for a student fellowship.

\section{References}

[1] Jacobson S C, Mcknight T E and Ramsey J M 1999 Anal. Chem. 71 4455-9

[2] Mensinger H, Richter Th, Hessel V, Döpper J and Ehrfeld W 1994 Proc. Micro Total Analysis Systems (Enschede, The Netherlands, November 21-22) pp 237-43
[3] Branebjerg J, Gravesen P, Krog J P and Nielsen C R 1996 Proc. IEEE Micro Electro Mechanical Systems 96 (San Diego, CA, February 11-15) pp 441-6

[4] Evans J, Liepmann D and Pisano A P 1997 Proc. IEEE Micro Electro Mechanical Systems 97 (Nagoya, Japan, January 26-30) pp 96-101

[5] Miyake R, Tsuzuki K, Takagi T and Imai K 1997 Proc. IEEE Micro Electro Mechanical Systems 97 (Nagoya, Japan, January 26-30) pp 102-7

[6] Fujii T, Hosokawa K, Shoji S, Yotsumoto A, Nojima T and Endo I 1998 Proc. Micro Total Analysis Systems 98 (Banff, Canada, October 13-16) pp 173-6

[7] Manz A, Bessoth F and Kopp M U 1998 Proc. Micro Total Analysis Systems (Banff, Canada, October 13-16) pp 235-40

[8] Schwesinger N, Frank T and Wurmus H 1996 J. Micromech. Microeng. $699-102$

[9] Brody J P, Yager P, Goldstein R E and Austin R H 1996 Biophys. J. 71 3430-41

[10] Burns M A et al 1998 Science 282 484-7

[11] Burns M A et al 1996 Proc. Natl Acad. Sci. 935556

[12] Sammarco T S 1999 PhD Dissertation University of Michigan, Ann Arbor

[13] Anderson R C, Bogdan G J, Barniv Z, Dawes T D, Winkler J and Roy K 1997 Proc. Transducers'97 pp 477-80

[14] Anderson R C, Bogdan G J, Puski A and Su X 1998 Proc. Micro Total Analysis Systems 98 (Banff, Canada, October 13-16) pp 11-6

[15] Anderson R C, Bogdan G J, Puski A and Su X 1998 Proc. Solid State Sensor and Actuator Workshop 98 (Hilton Head, SC, June 8-11) pp 7-10

[16] Hosokawa K, Fujii T and Endo I 1999 Anal. Chem. 71 4781-5

[17] Duda J L and Vrentas J S 1971 J. Fluid Mech. 45 247-60

[18] Bird R B, Stewart W E and Lightfoot E N 1960 Transport Phenomena 1st edn (New York: Wiley) p 62

[19] Bird R B, Stewart W E and Lightfoot E N 1960 Transport Phenomena 1st edn (New York: Wiley) p 559

[20] Cussler E L 1997 Diffusion Mass Transfer in Fluid Systems 2nd edn (Cambridge: Cambridge Univeristy Press) pp 81-6

[21] Taylor G 1953 Proc. R. Soc. 219 186-203

[22] Ottino J M 1980 Chem. Eng. Sci. 35 1377-91

[23] Ottino J M, Ranz W E and Macosko C W 1979 Chem. Eng. Sci. 34 877-90

[24] Carslaw H S and Jaeger J C 1959 Conduction of Heat in Solids 2nd edn (New York: Oxford University Press) p 101 